\title{
Development of a dynamic gravimetric calibration method for liquid water flow metering
}

\author{
Florestan Ogheard ${ }^{1, *}$ \\ ${ }^{1}$ CETIAT, Domaine scientifique de la Doua, 25 avenue des Arts, 69100 Villeurbanne Cedex, France
}

\begin{abstract}
According to its mission of national reference laboratory, LNECETIAT maintains and develops the French standard for liquid water flow meters calibration from $1 \mathrm{~g} \cdot \mathrm{h}^{-1}$ to $36 \mathrm{t} \cdot \mathrm{h}^{-1}$. The reference calibration facilities at LNE-CETIAT are historically based on the flying start and stop gravimetric method. Because of the specificity of micro-flow rates, two separated and dedicated calibration benches are used for flow rates from 1 g.h ${ }^{-1}$ to $10 \mathrm{~kg} \cdot \mathrm{h}^{-1}$ on one side, and from $8 \mathrm{~kg} . \mathrm{h}^{-1}$ to $36 \mathrm{t} \cdot \mathrm{h}^{-1}$ on the other side. Continuous improvement of the calibration capabilities pushes LNECETIAT to upgrade the existing methods toward a dynamic measurement of the reference flow rate, in the scope of EURAMET EMPIR "METROWAMET" JRP in which CETIAT is a partner of the consortium. After a short review of the state of the art and a description of the current LNE-CETIAT's, this paper will first present the development of a dynamic liquid flow generator required to ensure the measurand's representability. In a second part, the development of a dedicated and traceable measurement system, based on the existing gravimetric test rig, is described. Finally, the first feasibility test is presented and future developments are proposed.
\end{abstract}

\section{Introduction}

Calibration methods for liquid flowmeters have been widely presented and discussed in the literature since the beginning of flow metrology. While the diversity of approaches for such methods is real, the majority can be classified into one of the categories presented in the following table 1 and in [1].

In the literature about flow meters calibration methods, two interpretations of "dynamic" coexist. The first interpretation is in the sense of the measurement process: for example, dynamic weighing in the case of gravimetric methods. The second interpretation is in the sense of the flow properties fluctuations (flow rate, pressure, and temperature). Dynamic calibration methods for liquid flow meters have been extensively presented and discussed in literature and one can find detailed publications in the proceedings of the ISFFM and FLOMEKO conferences, coming mainly from NIST and PTB works.

${ }^{*}$ Corresponding author: florestan.ogheard@cetiat.fr 
The ideal dynamic weighing method for the calibration of liquid flow meters represents the natural evolution of gravimetric methods for several reasons:

- It reduces the calibration time

- It can be used for static (steady) and dynamic (fluctuating) flow conditions, thus ensuring comparability to historical static methods

- Representability of the calibration is maximized if the simulation of real-world flow fluctuation can be realized

- $\quad$ Direct traceability to mass and time standard is ensured

- Unlike volumetric methods, temperature-dependent (i.e. fluid density) measurement biases are minimized

- It allows for the characterization of other flowmeter's performances such as:

o The response time

o The stability of the error over a given (shorter) time interval

o The error against a defined flow profile

However, these ideal goals are yet to be reached (in particular being able to measure an instantaneous reference flow rate from a fluctuating flow with low uncertainties).

Table 1. Classification of liquid flow meters calibration methods. In green: the type of method covered by this article.

\begin{tabular}{|c|c|c|}
\hline & $\begin{array}{c}\text { Gravimetric } \\
\text { (weighing system) }\end{array}$ & $\begin{array}{c}\text { Volumetric } \\
\text { (scaled tank, provers) }\end{array}$ \\
\hline $\begin{array}{c}\text { Static } \\
\text { (Flying start-and-stop) }\end{array}$ & $\begin{array}{l}\text { Static weighing } \\
\quad+\text { diverter }\end{array}$ & $\begin{array}{l}\text { Scaled tank + } \\
\text { diverter, provers }\end{array}$ \\
\hline $\begin{array}{c}\text { Static } \\
\text { (Standing start-and- } \\
\text { stop) }\end{array}$ & Static weighing & Scaled tank, provers \\
\hline \multirow{2}{*}{ Dynamic } & ISO Definition & \multirow{2}{*}{$\begin{array}{l}\text { Dynamic level } \\
\text { gauging, provers }\end{array}$} \\
\hline & Dynamic weighing & \\
\hline
\end{tabular}

While each method has its own drawbacks and advantages, the present article focuses on the dynamic weighing calibration method, as described in the following. A review of dynamic gravimetric methods has been described in [2]. 


\section{LNE-CETIAT's liquid macro flow primary standard}

The French national standard for liquid (water) flow is held by LNE-CETIAT, which is the French Designated Institute for Liquid Water Flow, Humidity, and Air Speed. The main and historical calibration facility covers water flow rates from $8 \mathrm{~kg} \cdot \mathrm{h}^{-1}$ to $36000 \mathrm{~kg} \cdot \mathrm{h}^{-1}$, for a water temperature regulated from $15{ }^{\circ} \mathrm{C}$ to $90{ }^{\circ} \mathrm{C}$, and 1 to 3 bar relative pressure upstream of the flowmeter to be calibrated. The best relative calibration uncertainty is $5.10^{-4}(\mathrm{k}=2)$. The following figure presents the working principle of the macro-flow rates French primary standard.

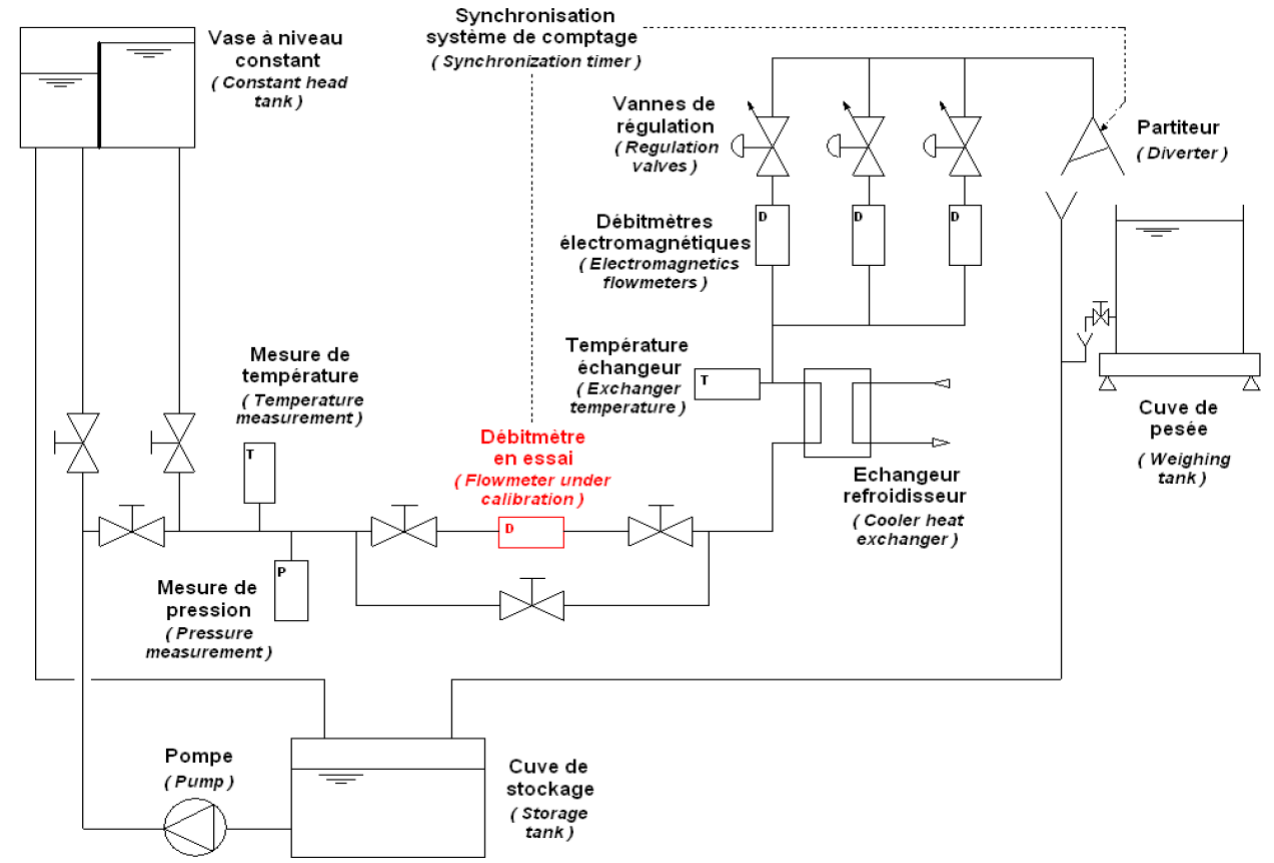

Fig. 1. French gravimetric primary standard for liquid flow held at LNE-CETIAT

The temperature regulated water in the storage tank is sent to the constant head tank which discharges at constant pressure (1 bar) through the flow meter under calibration. The liquid flows permanently through the flowmeter and is switched either to the weighing tank or to the storage tank.

Typically, for a pulse output flowmeter calibration, a first pulse generated by the flowmeter control the diverter switching to the weighing tank. At the moment of switch, a stopwatch and an external electronic counter used to obtain the number of pulses totalized are started simultaneously. After a certain amount of pulses set on the external counter and which corresponds to the filling of the weighing tank, the diverter switches back to the storage tank and both the stopwatch and the counter stop. 


\section{LNE-CETIAT's dynamic gravimetric liquid flow standard}

\subsection{Needs in the scope of Joint Research Project METROWAMET}

The METROWAMET project, funded under EURAMET EMPIR programme, aims at establishing a metrological infrastructure which will enable an integral characterization of domestic water meter performance close to real-world conditions and not at laboratory conditions as presently done [3].

Current test regimes for domestic water meters are prescribed to be run with well-defined, constant and reproducible reference flow rates. However, actual water consumption deviates strongly from these stationary conditions. This might have an impact on the cumulative uncertainty of meters which is not seen in the present standard continuous test regime. In consequence, meters might meet requirements at the test points but not the required cumulative uncertainty. So far there is no clear view on how the various types of domestic water meters actually perform under dynamic load changes as the metrological capabilities for this are missing at present. Furthermore, during the last decades the state of the art of water meters has progressed. Materials, fabrications have changed, but also meters based on new technologies are deployed. At the same time, the consumption behaviour of the consumers and the technical equipment in dwellings has changed significantly. The measurement trueness of water meters under these changed conditions still needs to be verified. In this project the metrological infrastructure required for this will be developed. Targeted conditions comprise dynamic load changes, water quality related aspects, and withdrawal of low amounts of water.

The work described in this article is within the scope of METROWAMET first work package. This work package deals with the development of the essential metrological infrastructure and protocols required to assess the performance of domestic water meters under a dynamic test regime in future instead of the current stationary one. Depending on the variability in consumer behaviour one or more pre-normative reference load profiles will be derived which will serve as input for the assessment of water meters under the novel test regime. Information about the representativeness of these profiles regarding region, season etc. will be provided. One additional result will be recommendations for the time-resolved monitoring of consumption profiles. Test rigs operated with dynamic load changes based on different principles will be realised and a comprehensive inter-comparison of the rigs and rigorous uncertainty assessment of the systems carried out. The rigs will meet at minimum the specifications of OIML R49 for the different applications for water meters given there. The following figure shows an example of dynamic flow profile against which water meters would be tested in this scope. 

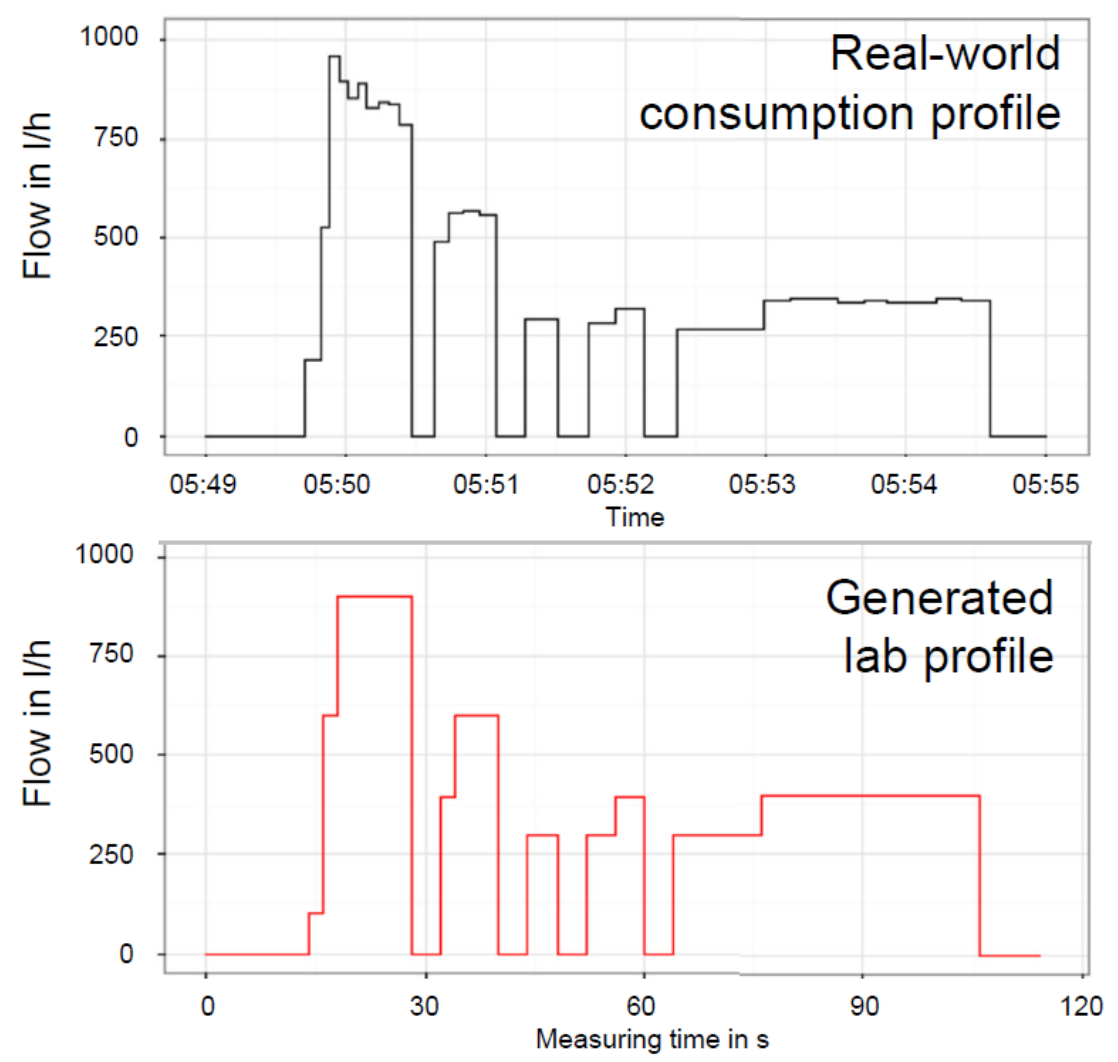

Fig. 2. Real world consumption profile and generated lab dynamic flow profile

\subsection{Development of the dynamic flow generator}

In order to cover the needs of JRP METROWAMET, LNE-CETIAT is developing a dedicated dynamic gravimetric test bench, which would act as a proof of concept to be duplicated onto the French liquid flow standard rig. The work focused first on the design of a dynamic flow generator able to generate fast fluctuating flow from $51 . \mathrm{h}^{-1}$ to $150001 . \mathrm{h}^{-1}$. The following figures 3 and 4 shows the schematic and 3D view of LNE-CETIAT dynamic liquid flow generator.

LNE-CETIAT's flow generator is composed of 3 flow settling closed loop lines, each dedicated to regulate of flow rate range of 1 to $5001 . \mathrm{h}^{-1}, 60$ to $18001 . \mathrm{h}^{-1}$, and 0.6 to $15 \mathrm{~m}^{3} / \mathrm{h}$. Each of these lines is composed of a flow meter and control valve, and can regulate a flow independently from each other. Consequently, to reproduce a flow profile such as shown in figure 2, a flow rate is generated, settled, then fed into the device under test line (see figure 3 ). During the feeding of a particular flow (a given plateau in figure 2), the dynamic flow generator is regulating the next flow step using one of the two unused line. To reproduce the flow profile, the flow generator system is switching from one line to another. With the chosen switching valve, flow switching from one plateau to the other is reached within one second. 


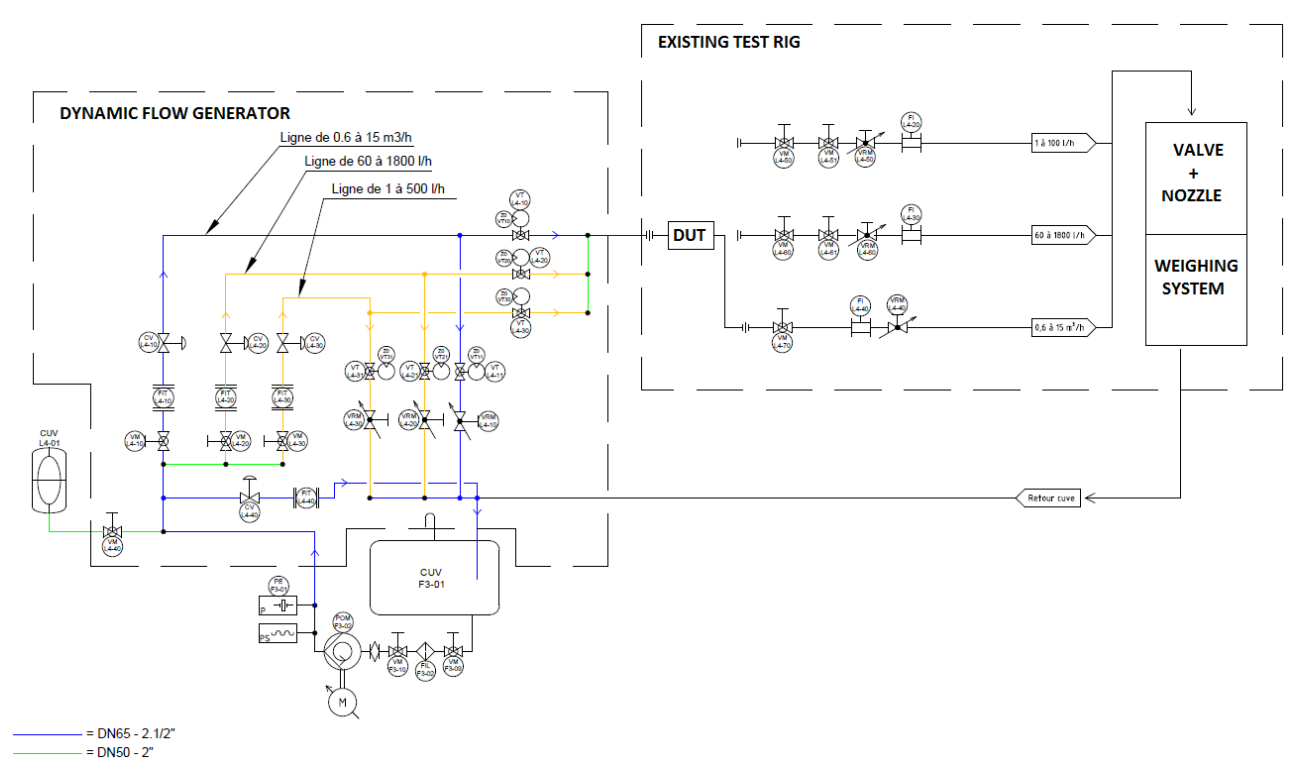

Fig. 3. Schematic of LNE-CETIAT dynamic liquid flow generator

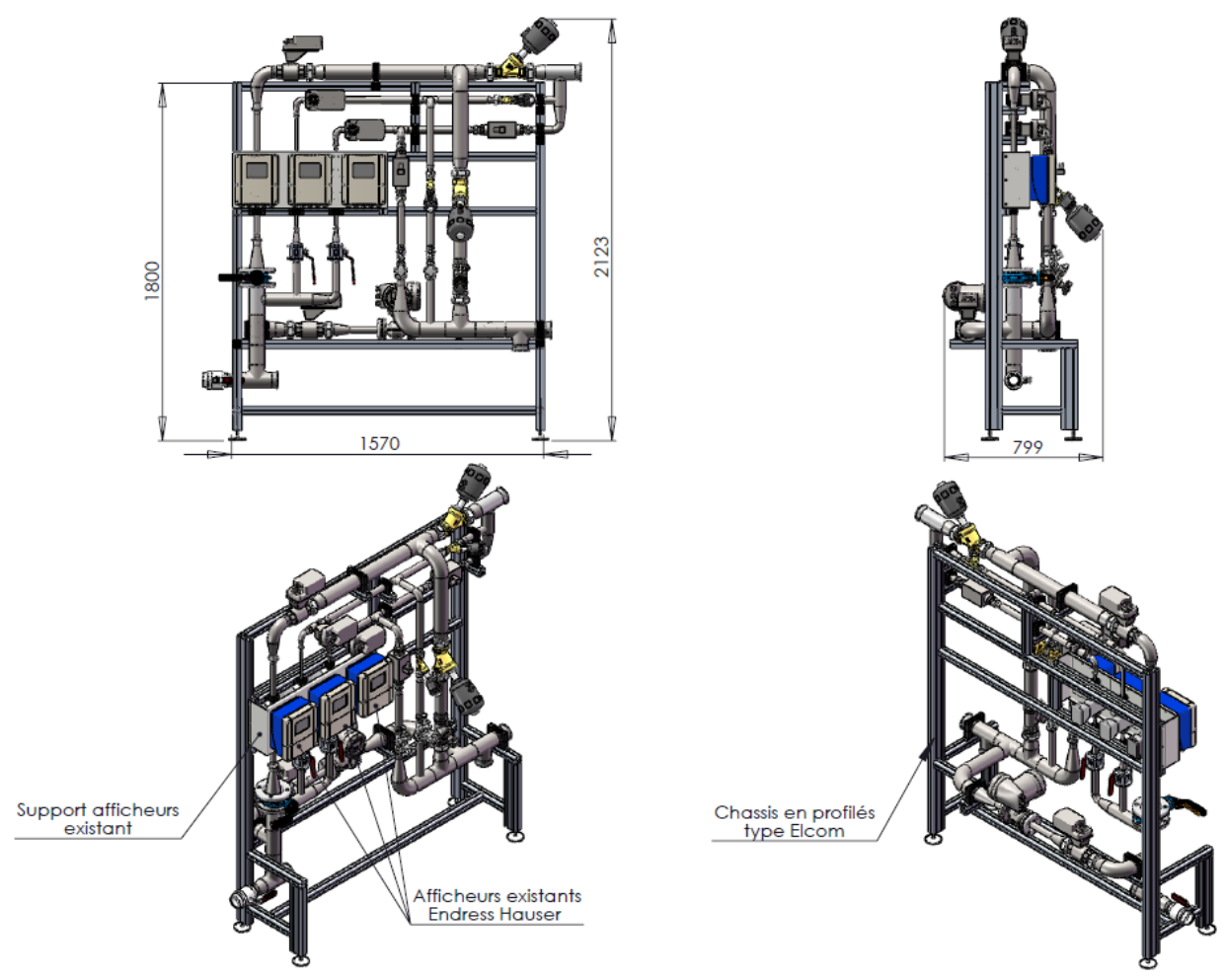

Fig. 4. 3D view of LNE-CETIAT dynamic liquid flow generator 
A dedicated software allows to program a given flow profile by entering each flow steps with given flow rate, duration and line number, such as presented in figure 5 below.

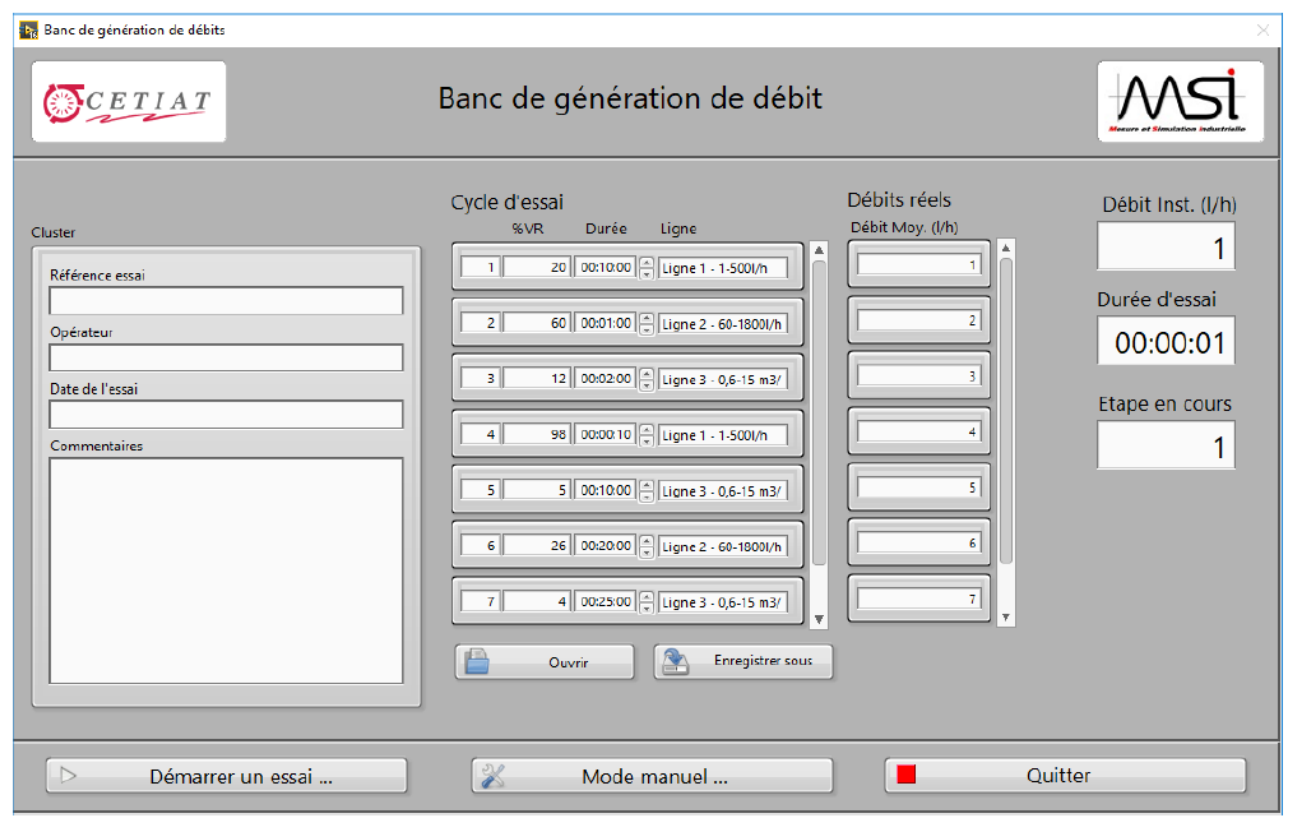

Fig. 5. User interface of LNE-CETIAT's dynamic flow profile generator

\subsection{Development of dynamic gravimetric liquid flow measurement system}

LNE-CETIAT's proof of concept dynamic gravimetric test rig is equipped with the same weighing scale as the French liquid flow standard. It is composed of a Sartorius IS150GG$\mathrm{HH}$ weighing scale, with a $150 \mathrm{~kg}$ range and a $1 \mathrm{~g}$ resolution. For the purpose of dynamic weighing, the weighing scale electronics has been upgraded with a YDO02C-AO 4-20 mA card. The figure 6 below shows the weighing system.

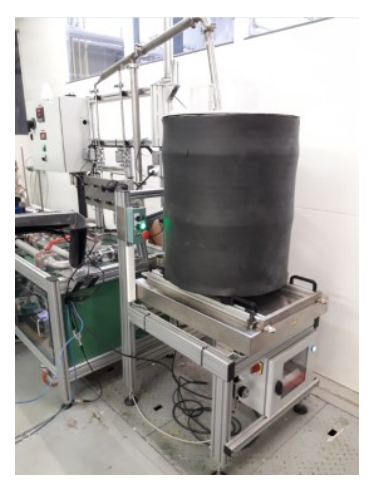

Fig. 6. LNE-CETIAT dynamic test rig weighing system 
In order to calculate the reference dynamic flow rate, the mass analog output is recorded at $1 \mathrm{kHz}$ sampling frequency using a GANTNER A101 24 bit analog to digital converter. The data processing required for the calculation of the reference mass flow rate is programmed using TEST.CON software from GANTNER. The diagram on figure 7 below shows the steps realized by the program.

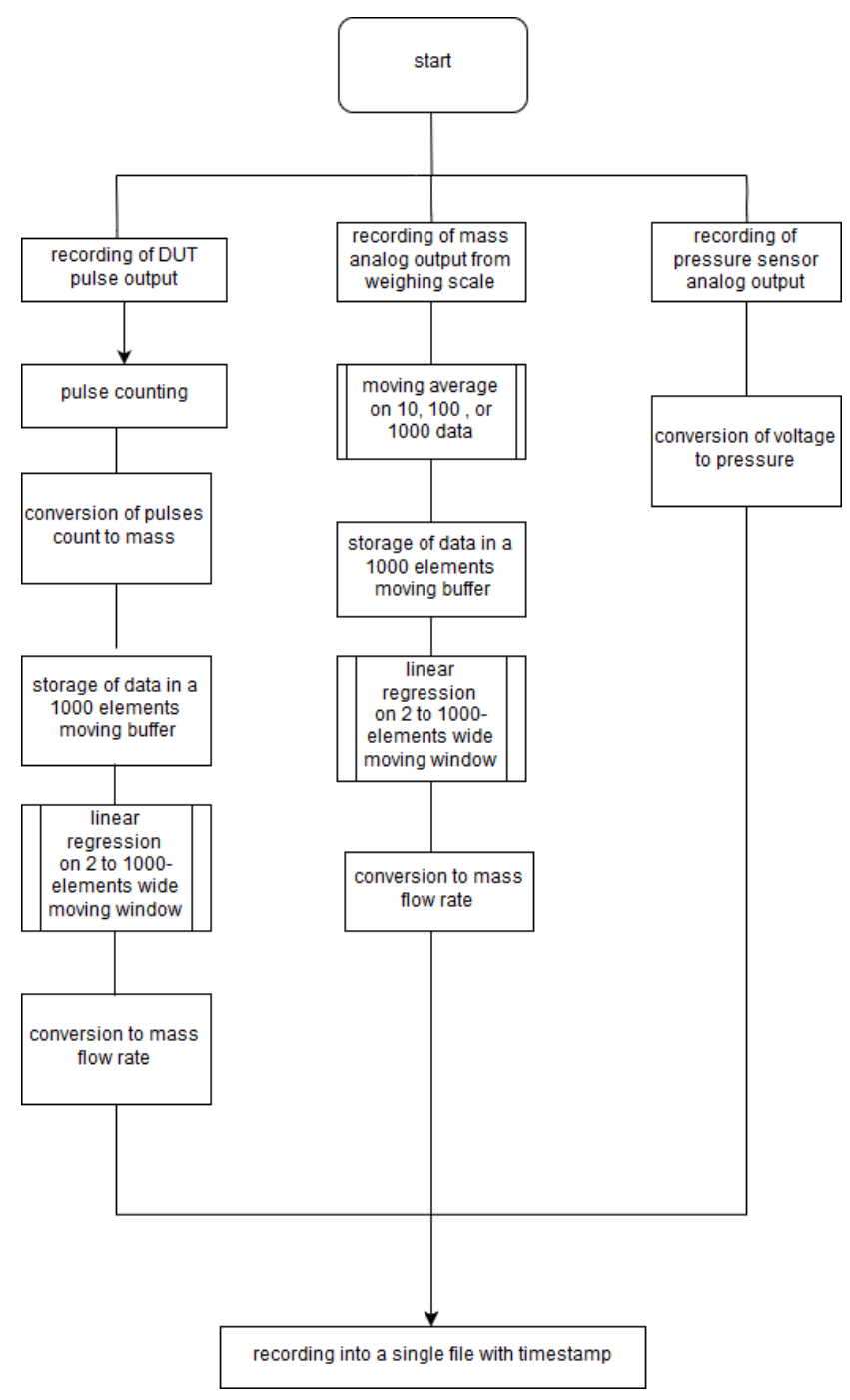

Fig. 7. Diagram of data processing

The data acquisition system associated with the data processing described in figure 7 allows for synchronous recording of the device under test (DUT) output, mass output from the weighing scale, and pressure sensor analog output from an in-line pressure sensor downstream of the DUT. 


\section{First test of dynamic gravimetric flow measurements and future work}

\subsection{First qualitative test of dynamic flow profile generation and measurements}

First tests of the dynamic gravimetric flow test rig has been realised with a Emerson MicroMotion Elite CMF025 Coriolis mass flow meter as the device under test. Its pulse output has been set to a pulse weight of $0.01 \mathrm{~g}$. The following figure 8 shows raw data, acquired synchronously by the system described in 3.3 for a dynamic flow profile generated by the system described in 3.2 .

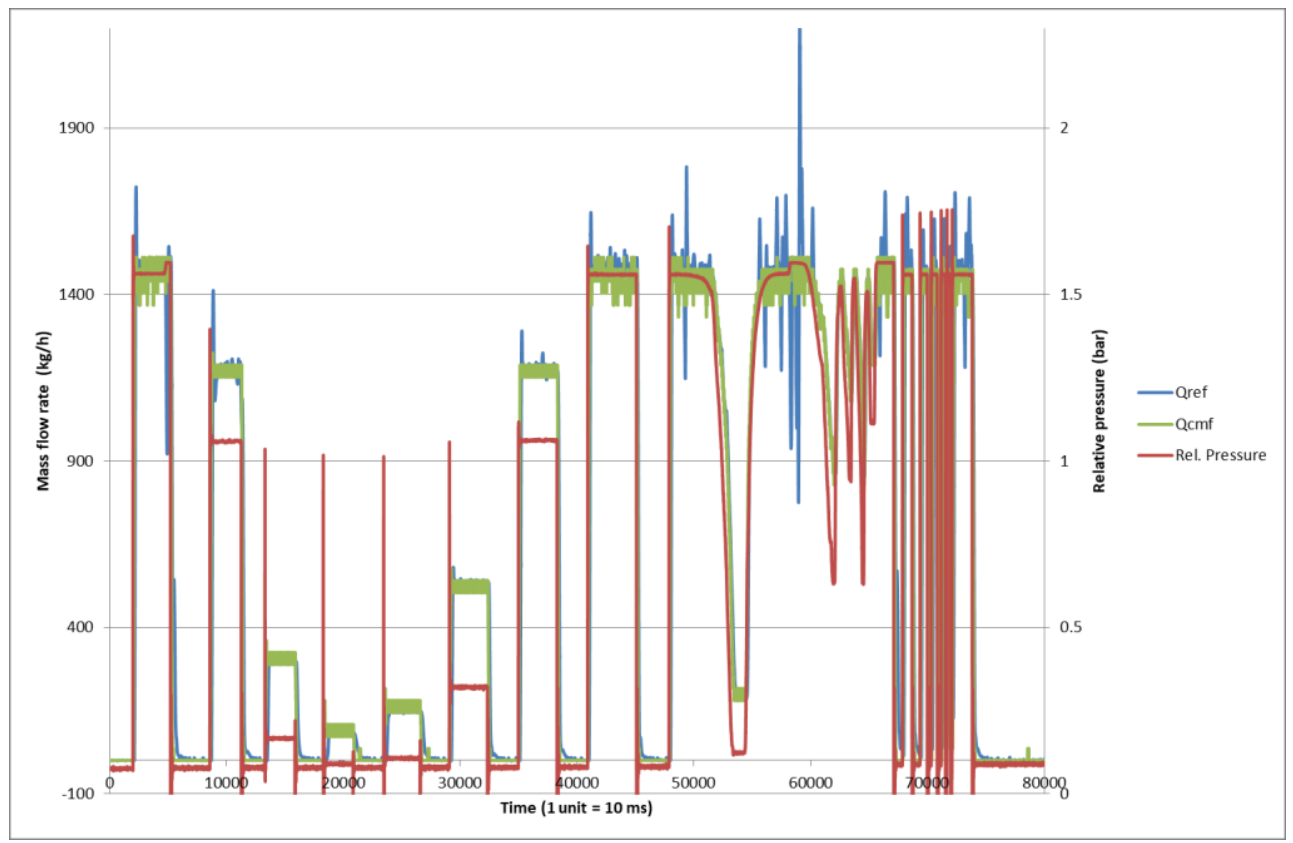

Fig. 8. Raw data acquired for reference mass flow (blue), DUT mass flow (green) and relative pressure downstream of the DUT (red)

The generated flow profile was used to check the feasibility to generate flow steps, ramps and fluctuations with simultaneous recording, of reference mass flow rate, pressure, and DUT output. Despite showing good capability to follow real time flow fluctuations, more work is required to filter raw data and improve accuracy. Measurement noise on mass data, as shown in figure 8 , has to be filtered if not representative to real flow rate fluctuations. Inline pressure, as recorded synchronously, is a reliable indicator of flow variations. Quantitative assessments and validation, undergoing at LNE-CETIAT, will be presented in future publications. 


\subsection{Ongoing and future work}

Current work focuses on the data processing required to measure accurate mass flow rate, without degrading its time response relative to the DUT to assess. Indeed, filtering, from moving average to more complex data processing, requires time that add delay in the processing of reference mass flow rate. To reach such goal, LNE-CETIAT is developing an algorithm, based on the diagram shown in figure 7, which will adapt its filtering degree depending on mass and pressure inputs fluctuations. Indeed, a mean to detect if mass fluctuations are due to real mass flow rate fluctuations is to calculate correlations between mass and pressure. This can be done in quasi real-time, and correlation coefficient can be fed into the filtering algorithm, or used in post-processing. Examples of filtering mass data for dynamic gravimetric flow rate measurements can be found in [4].

In the scope of JRP METROWAMET, LNE-CETIAT will take part, in summer 2020, in an international comparison of National Metrology Institutes and Designated Institutes, all developing dynamic flow calibrations facilities, to validate the methods developed, and submit new Calibration and Measurements Capabilities (CMCs) to the Bureau International des Poids et Mesures (BIPM) Key-Comparisons Data Base (KCDB). The transfert standards will be provided by LNE-CETIAT and will be based on an Emerson MicroMotion Elite CMF025 Coriolis mass flow meter.

Consequently, the dynamic gravimetric test rig will be used to assess water meters and more generally liquid flow meters, using methodology such as provided in [5,6]. This will allow providing more representative calibrations to customers with complementary and useful information such as time response and accuracy against defined flow profiles and transients.

\section{Conclusion}

In the scope of JRP METROWAMET, and in order to develop more representative primary calibration methods for water meters and more generally liquid flow meters, LNE-CETIAT is developing a dynamic gravimetric calibration test bench. It is composed of a dynamic flow profile generator, and a gravimetric system. Data acquisition allows synchronous recording of reference mass flow, device under test output and in-line pressure. Feasibility of generating and measuring dynamic flow profiles has been demonstrated. Finally, ongoing and future work focuses on data processing in order to provide accurate assessment of liquid flow meters under real-world flow conditions.

\section{Acknowledgment}

The EMPIR project "METROWAMET" is carried out with funding of European Union under the EMPIR. The EMPIR is jointly funded by the EMPIR participating countries within EURAMET and the European Union. 


\section{References}

1. R. Engel, Aspects of traceability and comparisons in flow measurement, Proceedings of the 7th ISFFM, Alaska, USA, August 12th-14 ${ }^{\text {th }}$ (2009)

2. F. Ogheard, First steps toward dynamic reference calibration methods for liquid flow meters at LNE-CETIAT, $10^{\text {th }}$ ISFFM, Querétaro, Mexico (2018)

3. https://www.ptb.de/empir2018/metrowamet/the-project/

4. J.J. Aguilera Mena, Dynamic weighing calibration method for liquid flowmeters - A new approach, Thesis dissertation, Braunschweig, (2012)

5. D. Wiklund and M. Peluso, Flowmeter dynamic response characteristics Part 1: quantifying dynamic response, Rosemount, Inc., Chanhassen, MN (2002)

6. D. Wiklund and M. Peluso, Flowmeter dynamic response characteristics Part 1: effects in various flow applications, Rosemount, Inc., Chanhassen, MN (2002) 